\title{
Mechanism Research on Treatment Measures Regarding the Branching Reach with Bend at the Middle and Lower Yangtze River after Water Storage of Three Gorges Reservoir

\author{
Yude Zhu ${ }^{1, a}$
}

${ }^{1}$ Tianjin Water Transport Engineering Science Institute, Ministry of Transport, Tianjin China 300456 a Zhuyude2800@sina.com, ${ }^{\mathrm{b}} 22845474 @ q q . c o m$

Key Words: Water storage; Measures; Mechanism; Branching reach with bend;

\begin{abstract}
This paper analyzes the characteristics of the flood land change at the branching reach with bend after water storage of Three Gorges of Yangtze River, and demonstrates in combination with numerous engineering practice researches that the treatment measures regarding the branching reach with bend at the middle reaches after water storage of Three Gorges are significantly different from the traditional treatment measures, and puts forward reasonable theoretical supports for such treatment measures after discussion, so as to realize the increase of channel dimension via controlled floodland.
\end{abstract}

1. Characteristics of the changes of the branching reach with bend at the middle Yangtze River after water storage of Three Gorges

There are various river patterns distributed in the middle reaches of Yangtze River. Among others, the river patter of branching reach with bend exists in an extensive range. Such rivers are normally larger in river width, with the local main stream at the branching reach liable to shift, and relatively weak characteristics of the water flow movement, which leads to the tendency of the formation of various floodlands; in the meantime, there are certain limitations to the river width, as a result, despite the existence of various floodlands, such floodlands exist mostly in form of point bars or central bars rather than grow high. Normally, point bars are attached to one bank, usually located in areas of slack water, and distributed on the river channel side or the convex bank of the bend at the straight branching reach, with close or semi-isolated connection between the floodland and the river bank, such as Nianziwan point bar, Chaimatou point bar, Yanggouzi point bar and so on; central bars are relatively independent underwater disposition bodies, some of which are relatively stable while others are not; stable central bars are normally located in non-mainstream area, low and gentle and relatively complete, while the central bars located in the mainstream fluctuating area with greater water flow changes of floods and droughts are normally unstable, such as Ouchikou central bar and so on.

According to the statistics, the shallow rate of the branching reach with bend with width of $1500 \mathrm{~m} \sim 2500 \mathrm{~m}$ at the middle reaches of Yangtze River after water storage of Three Gorges increases significantly, compared with that before water storage, which is mainly because that the existing low floodlands of the original waterway at such reach is prone to undergo scour under the circumstance that the water duration increases greatly while the coming sediment decreases significantly after water storage of Three Gorges; the floodland restoration capability of the floodland after it is reduced to the floodland and the reach after scour presents a significant development tendency of widening and shallowing,thus the situation of worse channel conditions appears. 
2. Treatment of branching reach with bend at the middle reaches of Yangtze River after water storage of Three Gorges

Treatment measures regarding the reach existing in the form of "point bar"

Point bars exist in both straight branching reach and branching reach with bend, usually attached to one bank as floodlands with the same direction as or a small angle to the water flow direction. The reorganization of the treatment regarding the branching reach with bend existing in the form of point pars at the middle reaches of Yangtze River after water storage of Three Gorges reveals that the treatment of such river pattern performs protection to the point bars mainly by adopting the flexible mattress beach protection belt, which is relatively well-set and characterized by transformation within a certain range along with floodland. Different point bar protection patterns are slightly different in that arrangement of flexible mattress beach protection belt of banded interrupted protection pattern is usually adopted with respect to the reach with great lateral mainstream fluctuating and floodland transformation focusing on lateral erosion, while overall protection may be performed to the floodland if necessary with respect to the major reach with complex flow state and great flow rate, and overall protection will be performed to the scouring complex area in accordance with relevant experiment and research with regard to the plane, so as to keep adequate impact strength; a method combining centralized protection and interrupted protection are normally adopted to the same floodland at the same location subject to different water flow conditions.

Point bar protection adopting flexible mattress beach protection belt, especially the form of banded protection, is rather intensively applied in the middle reaches of Yangtze River, with significant effect. Since the water storage of Three Gorges, undesirable changes such as scouring, moving down and so on occurred to the left boarder of Ouchikou central bar, the beachhead of the Daoxinyao central bar, and pointed bar of Tuoyangshu; the water flow fluctuation is great and the borders of floodland and navigation channel are unstable; in order to form and stabilize favorable floodplain and main channel pattern, the treatment of Ouchikou waterway and navigation channel adopts the performance of protection engineering to Tuoyangshu point bar (fig.1). Similarly, the Guniusha point bar on the right side waterway is gradually washed away, the elevation of floodland surface is reduced, the floods and droughts areas at the branching reach increase, and the navigation conditions deteriorate as a result of the Guniusha waterway under the influence of lessened coming sediment, changing incoming water flow, floodland sand excavation and so on after water storage of Three Gorges, banded interrupted beach protection belt is performed to the right point bar focusing on lateral erosion in the phase I project (fig.1), which stabilizes the state of Guniusha point bar and prohibits the deterioration of the navigation conditions.

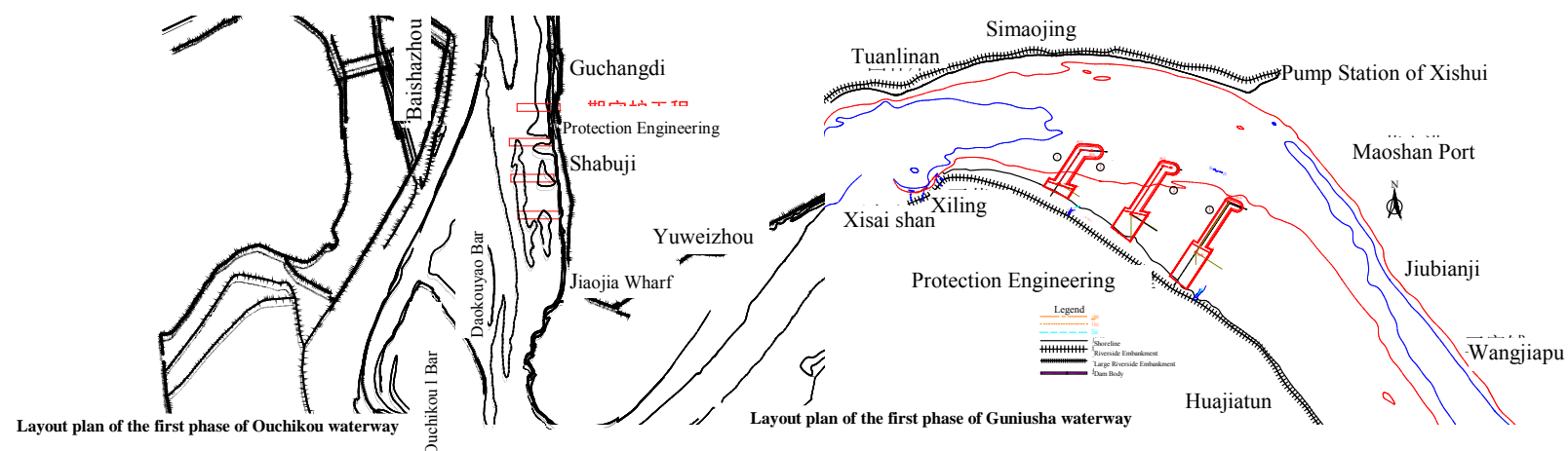

Fig. 1 Layout of Protection of Reaches Existing in the Form of "Point Bar" at the Middle Reaches of Yangtze River

\section{Treatment measures regarding the reach existing in the form of "central bar"}

Central bars are relatively independent underwater disposition bodies, which are basically characterized by that they are normally located in branching waterways, with some relatively stable while others not; stable central bars are normally located in non-mainstream area, low and gentle and relatively complete such as the Laohutan of the east-flow waterway of Yangtze River, while the 
central bars located in the mainstream fluctuating area with greater water flow changes of floods and droughts are normally unstable, such as Ouchikou central bar. Ouchikou central bar belongs to the straight transitional shallows at the lower reaches of the division port. Under the influence of mainstream fluctuation and the growth and decline changes of the central bar in the upper reaches, both the left and right flumes of the central bar are unstable, and prone to shallow during the process of trough fluctuation and translocation.

Fish mouth type beach protection belt is usually adopted in most cases with regard to the branching reach with bend existing in the form of central bar in the middle reaches of Yangtze River after water storage of Three Gorges, to perform defensive protection to the river central floodland (bar), stabilize the river head, and prevent the river central floodland (bar) from being washed lower or away. Take Liyushan waterway in the middle reaches (fig. 2) for example. After water storage of Three Gorges, as the point bar on the right bank is divided and forms Huanglianzhou central bar, and the branching reach forms the pattern of south and north drought passages, the south passage scouring depth develops rapidly while the north passage shallows, and the navigation conditions increase in number and get worsened during the deposition process of relatively small Huanglianzhou; in order to cope with the channel changes, the treatment project to be implemented adopts the fish mouth type beach protection measures to protect Huanglianzhou, through the treatment idea of appropriately intensifying the drought water flow strength in the shallow area and improving the navigation conditions in the shallow area.

The treatment of the river head lower floodlands of some divided reaches after water storage of Three Gorges adopts offensive protection by applying low straight dam at the river head on the basis of fish mouth type beach protection belt, so as to connect the scattered river central floodlands (bars) to form a relatively integrate floodland state, and play a role of intercepting cross current and adjusting water flow direction. For example, the river head of Daijiazhou reach (fig. 2) enters into the development stage of slump scour after water storage of Three Gorges, which is undesirable to both the straight channel and the round channel; in the meantime, after the application of the water storage of Three Gorges, the floodland restoration rate of the higher floodland after scouring will be significantly weakened; in order to prevent the adverse reach changes to be caused by continuous scour of the river head, the current relatively favorable opportunity shall be made use of, to perform river head protection project in phase I, effectively improve the navigation conditions at the entry transitional channel and restrict further scour of the river head.

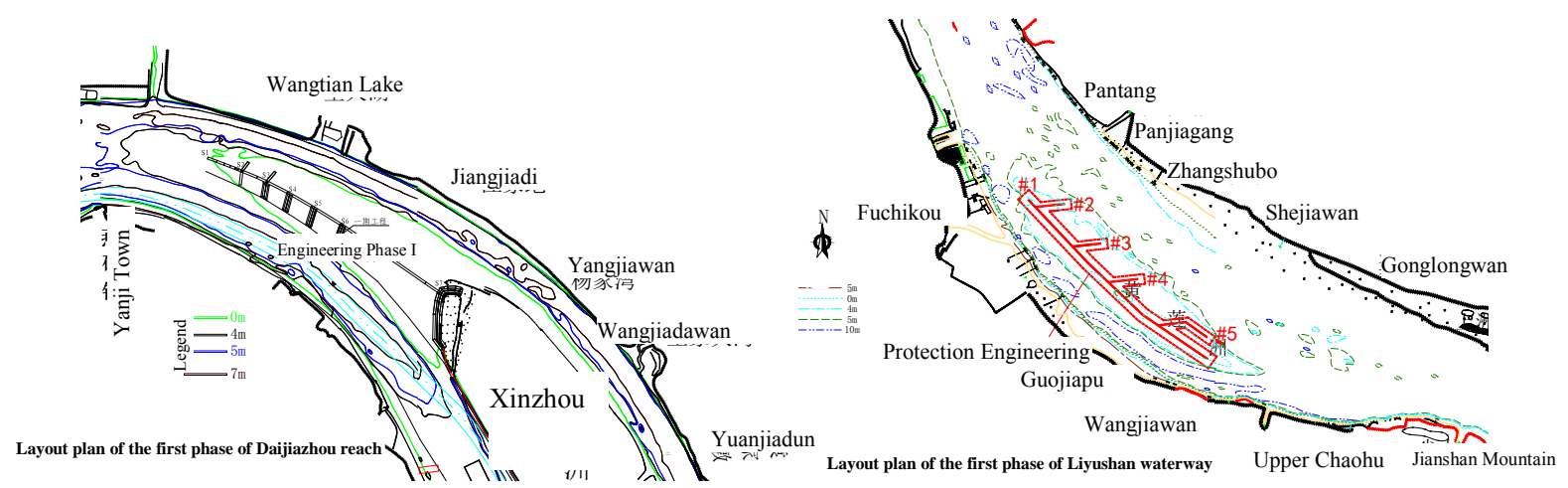

Fig.2 Layout of the Protection Project of Reaches Existing in the Form of Central Bar at the Middle Reaches of Yangtze River

4. Analysis of the difference between the treatment measures regarding the branching reach with bend at the middle and lower reaches of Yangtze River before and after water storage of Three Gorges

The channel treatment engineering implemented and to be implemented to the middle and lower reaches of Yangtze Rivers after water storage of Three Gorges performs necessary protection and control to the floodland to which certain adverse changes occurred in the earlier stage of water storage of Three Gorges. As shown by the treatment effects, after the implementation of protection 
engineering, favorable floodplain form of multiple navigation-obstructing shoals obtains a certain degree of protection, and the channel conditions are significantly improved.

Natural navigational alluvial rivers usually increase the channel width by constructing higher spur dike, longitudinal dike or other regulating structures, realizing channel dimension increase through clearing sands with converging flow. The principle thereof is to increase the navigational water depth of the section by breaking the balance relationship between the original channel section width and the channel depth of the reach and reducing the effective drought width of the section, and to reach a new kind of balance after regulation for a period.

The practice of channel regulation indicates that the channel treatment of the middle and lower reaches of Yangtze River after water storage of Three Gorges is mostly realized by controlling favorable floodland form through low beach protection structures, which effectively increases the planed channel dimension within a certain range. After the protection of the favorable floodland situation, the floodland built by the river flow itself is able to comply with the characteristics of the water flow movement, give play to the water flow flute and furthermore improve the channel conditions. However, there is still a lack of complete theoretical statement of the dynamic mechanism and internal causes of the current river control project in realizing the improvement of the channel conditions.

5. Mechanism analysis of the treatment measures of controlling the floodland at the middle and lower reaches of Yangtze River after water storage of Three Gorges

After the completion of Three Gorges, under the influence of storage peak to adjust the drought, clear water release and so on of the reservoir, the incoming sediment conditions of the reach at the middle and lower Yangtze River change significantly; the original relative balance is broken, and a process of riverbed reconstruction and balance reestablishment is entered. The traditional treatment measures of clearing sands with converging flow is to break the original balance and then establish a new balance through offensive engineering, while the original balance of Three Gorges has been broken under the condition of clear water release, and the reservoir releases clear water or sub-saturated water flow; therefore, the sediment content supply along the river is necessary to transit from sub-saturation to saturation, so as to form a new balance, and entire reach scour will occur to the riverbed in the lower reaches. Consequently, the only demand is to guide such clear water to scour the desired area and protect the floodland not expecting scouring, which is what we proposed to obtain larger channel dimension through controlling project with the purpose of protecting the floodland under the condition of clear water release.

According to the scouring balance below the dam of the plain tract, the scour must firstly realize the bed material armoring but not hanging, thus the scouring balance of bed load must be satisfied. After substitution into the continuity equation of flow, it can be derived that to re-establish the scouring balance below the dam, the river bed must satisfy $Q / B h^{7 / 6} \leq K^{\prime} D^{1 / 3}$, in which, river width $b$, average water depth $\mathrm{h}$, and grain size of bed material $\mathrm{d}$ are under continuous adjustment. During such adjustment process, in order to achieve the purpose of increasing water depth $h$, we may control the changing range of the river width and the grain size of the bed material through engineering measure, while the control of river width changes is the most effective and most achievable measure. Under the circumstance of controlling river width changes, the clear water continuously deepens the river bed through scouring, and the river bed material is also continuously coarsened, until the scouring stops and a new balance is reached. With respect to the reach at the middle and lower Yangtze River, we can definitely achieve the purpose of increasing the water depth to a certain degree only by restricting the waterway from lateral extension, that is, to prevent the low floodland in the waterway from be scoured and thus make the clear water scouring can only develop in the flume along the longitudinal direction; this is also the major mechanism to realize water depth increase by focusing on guardian engineering. 


\section{Conclusion}

Under the circumstances that the water duration increases while the coming sediment from the upper reaches decreases with regard to the branching reach with bend at the middle reaches of Yangtze River after water storage of Three Gorges, the floodland restoration capability of the floodland after the floodland scouring decreases greatly, and the presents a significant development tendency of widening and shallowing,

Under the normal operation conditions of Three Gorges, the treatment of the branching reach with bend at the middle reaches of Yangtze River realizes the purpose of channel regulation mostly through protecting and controlling favorable floodland forms, whose treatment mechanism is significantly different from the treatment of the natural reach; however, seen from the regulation effects, after the implementation of protection engineering, the channel conditions are significantly improved.

As found in the research, during the process of river bed reconstruction and the balance re-establishment at the lower reaches of the hub, the river bed must satisfy $Q / B h^{7 / 6} \leq K^{\prime} D^{1 / 3}$; during the adjustment process, in order to achieve the purpose of increasing water depth $h$, we may control the river width through engineering measures. We can definitely achieve the purpose of increasing the water depth to a certain degree only by restricting the waterway from lateral extension and thus make the clear water scouring can only develop in the flume along the longitudinal direction.

\section{References:}

[1] Zhu Yude. A Experimental Study of the Fixed Bed Physical Model of the Channel Treatment Engineering Regarding Liyushan Waterway at the Middle Reaches of Yangtze River [R]. Tianjin,Tianjin Research Institute for Water Transport Engineering of Ministry of Communications and Transportation, 2013.

[2] Zhu Yude;.Influencing Factors of Shoal Evolution and Regulation of Xinzhou Confluence in the Middle Yangtze River [J].Port \& Waterway Engineering, 2012 (8): 124-128.

[3] Liu Wanli, Et. Al . Study on Channel Regulation of Daijiazhou Reach in Middle Yangtze River [J]. Journal of Waterway and Harbor, 2009 (6): 418-424.

[4] Zhu Yude. Discussion on Waterway Regulation of Shashi Section at the Midstream of the Yangtze River [J]. Journal of Waterway and Harbor, 2006 (5): 223-226. 